\title{
Tailor-made poisons for pathogens
}

programmed
Cas9 is
sufficient for
mediating
sequence-
specific
cytotoxicity of
resistant cells

A major drawback of traditional antibiotics is their inability to specifically target and destroy bacterial pathogens, which results in the undesirable killing of beneficial bacteria and promotes the emergence of resistance. Two studies now report the use of CRISPR-Cas (clustered, regularly interspaced short palindromic repeats-CRISPR-associated proteins) technology for the creation of customizable, sequence-specific antimicrobials that target antibioticresistant bacteria only.

Cas9 is a double-stranded DNA (dsDNA) nuclease from the bacterial type II CRISPR-Cas system and it can be programmed to cleave almost any desired DNA sequence through the use of short, guide RNAs that specify the cleavage site. Bikard et al. and Citorik et al. began by transforming Staphylococcus aureus and Escherichia coli, respectively, with plasmids that encoded Cas9 and $\lambda$ guide RNAs, to specifically cleave a chromosomally encoded resistance gene (the aph gene encoding kanamycin resistance in S. aureus) and one of two different $\beta$-lactamase genes (the $b l a_{\mathrm{NDM}-1}$ or $b l a_{\mathrm{SHV}-18}$ genes encoding $\beta$-lactam resistance in E. coli). Both groups showed that a substantially lower number of transformants were recovered from cells that encoded the resistance genes compared with susceptible cells, which confirms that programmed Cas9 is sufficient for mediating sequence-specific cytotoxicity of resistant cells.

To use Cas9 as an antimicrobial requires an efficient delivery system for bacteria in natural environments. As phage are natural predators of bacteria, both groups created phagemids (which are plasmids that are packaged into phage capsids) encoding the Cas9 nuclease and the guide RNAs that target $a p h$ and $b l a_{\mathrm{NDM}-1}$ or $b l a_{\mathrm{SHV}-18}$. In each case, phagemid-mediated delivery of the customized Cas9 resulted in the killing of those cells that encoded the cognate resistance genes, whereas cells that lacked these genes survived. In addition, Citorik et al. found that a customized guide RNA was capable of discriminating between sensitive and resistant strains that differed by a single-nucleotide mutation in the DNA gyrase gene gyrA, which confers resistance to quinolone antibiotics. Furthermore, although both groups found that a fraction of targeted cells escape killing, Bikard et al. showed that the Cas9 system is nevertheless advantageous over antibiotics as it enables non-targeted bacteria to continue to grow and thereby outcompete resistant cells.

Antibiotic resistance genes are often present on plasmids that are readily transferred between bacteria, and both teams showed that the Cas9 system can also be used to destroy such plasmids, thereby restoring antibiotic sensitivity to a bacterial population. Moreover, Bikard et al. showed that treatment of plasmidfree cells with programmed Cas9 results in immunization against plasmid transfer, so the system also prevents the spread of plasmid-borne resistance. Another advantage of the Cas9 system is the possibility of a multiplex approach for the targeting of more than one resistance and/or other virulence genes using several guide RNAs. Citorik et al. showed that simultaneous targeting of both the $b l a_{\mathrm{NDM}-1}$ and $b l a_{\mathrm{SHV}-18}$ genes was possible in E. coli, and Bikard et al. created a phagemid that targeted both the methicillin resistance gene mecA and the sek gene, which encodes an enterotoxin in S. aureus.

Finally, both groups demonstrated that customized Cas9 antimicrobials selectively kill bacterial pathogens in vivo. Citorik et al. showed that treatment with a phagemid designed to kill enterohaemorrhagic E. coli by targeting the virulence factor intimin resulted in increased survival of infected Galleria mellonella moths compared with untreated controls. Similarly, Bikard et al. used a mouse skin colonization model to show that customized Cas9 resulted in the selective killing of kanamycinresistant $S$. aureus.

Although these studies show that Cas9 antimicrobials have several advantages over traditional antibiotics, owing to caveats associated with phagemids (such as phage resistance and limited host-range), other delivery vehicles, such as polymeric nanoparticles, will need to be explored for the system to reach its full potential as a versatile, but selective, antimicrobial.

Christina Tobin Kåhrström

ORIGINAL RESEARCH PAPERS Bikard, D. et al. Exploiting CRISPR-Cas nucleases to produce sequence-specific antimicrobials. Nature Biotechnol. http://dx.doi.org/10.1038/nbt.3043 (2014) |Citorik, R. J., Mimee, M. \& Lu, T. K. Sequence-specific antimicrobials using efficiently delivered RNA-guided nucleases. Nature Biotechnol. http://dx.doi.org/10.1038/nbt.3011 (2014)

FURTHER READING van der Oost, J. et al. Unravelling the structural and mechanistic basis of CRISPR-Cas systems. Nature Rev. Microbiol. 12, 479-492 (2014) 